\title{
African textile design and fabric arts as a source for contemporary fashion trends
}

\author{
Dereje Debeli $^{1, a}$, Liu Jie Yuan ${ }^{1, b}$, Zhou Jiu ${ }^{1, c_{*}}$ \\ ${ }^{1}$ Key Laboratory of Advanced Textile Materials and Manufacturing Technology, Ministry of \\ Education, Zhejiang Sci-Tech University, Hangzhou, Zhejiang, 310018, China. \\ adrjdebeli@gmail.com, bgaara.gaara@qq.com, 'chhoujiu34@126.com
}

Key words: African textiles, design, fabric, arts

\begin{abstract}
Textile design and arts in Africa traced back to thousand of centuries when some ethnic groups were started producing clothes for multi-purpose uses. Their design creations and arts were known to be having geometric patterns graced with wonderful choice of colors which were primarily bright in that sense. This paper introduces African textile designs and dressing styles. The concept of designing African textiles and its method to integrate on contemporary fashion trends are intended to be discussed. Color choices, selection of patterns and combination of aesthetics values are also included after taking some examples that illustrated African textiles. The kind of motif selection, balancing shapes and color sonority imbedded on these fabrics have a lot of thing to do with their wearing culture and dressing styles. The import of motifs on fabric has its own techniques. Textile design and fabrics produced based on these techniques are finally planned to convey known values from generation to generation.
\end{abstract}

\section{Introduction}

African textile designs and arts are representative symbols of African society. Some people and societies in Africa have developed symbols from proverbs, sayings, and king's quotation in a form of arts. Similarly, other ethnic groups also use the cultural landscapes, natural objects, or their innovative works in a form of arts for their contemporary designs. It is these symbols that have been collected from various ethnic groups and then presented artistically through patterns for fabric decoration and design. Later these design elements transformed and embedded on fabrics. The aesthetics value of these African textile designs have well defined in a form of design elements such as lines, curves, symbols or motifs.

Basically, African textiles design originated from the cultural backgrounds of 55 African countries and more than 800 linguistic groups. The continent has diverse physical environments ranges from mountains to arid deserts, Great Rift Valley and rain forests. These entire physical environments will determine what African people can design and dress beside the influence of missionaries and explorers. These cultural and environmental differences did not bring a difference, rather contributed for the foundation of flame buoyant styles, beautiful cultural dressing, wonderful fine arts and traditional crafts of present day African textiles [1, 2].

The arts and aesthetics of African textile has been appreciated by a lot of scholars and used as inspiration for their works. Upon getting motivation from African arts, Picasso had step up his works and modernizing western arts through African touched inspiration. According to Picasso, most present day western arts were sourced from African origin and he had started a revolution on western arts. Matisse also had discovered that African textile arts were embedded uncovered abstract on its geometric appearance that thought to bring aesthetics value. Raffia design style of Congo people has the main input for his studio decoration and carpet design ever appreciated in his design collection. Designers such as Modigliani and Giacometti were also fascinated by African design style, decoration and selection principles that have helped them for collection and developing an extraordinary textile design and fashion appreciated in their ages [11, 12]. These designers and artist enthusiastically impressed by the aesthetic value of African arts, motifs and patterns for getting idea. The symbolic representation and its techniques are very fascinating for the 
scholars. Later, the extraordinary legacy of these arts with its explosive color and complex graphic patterns has been used as source of inspiration or direct input of their design.

This paper forwards the fabric design principles based on African dressing styles. The design concept is borrowed from African designs. For this case, given patterns were selected as it to represent African textile pattern. So, from this study, concept of design, color combining styles and system to produce on jacquard weave can be introduced. These steps and design principles can be also used as an input for other researchers. It doesn't matter whether we use the design for weaving or printing purpose. Patterns produced following African design principle stands to represent natural or artificial objects in a form of complete design.

\section{Design concept}

There are various ways to design patterns from different ethnic symbols mentioned earlier. To develop design based on African style under given condition seams easy, but this is not always true. Collecting information such as, type of motifs, power full patterns and coloring styles along its basic usage are things to be considered. The color selection and its combination on pattern is a bit hard [6]. Which can be obtained either through contrastive colors (hot and cool of equal strength) or by maintain the equality of dynamics in phrasing of lightness and darkness in colors. Thus, this selection must be in line with chosen type of usage, like as ceremonial clothes, daily based dresses or other purposes in order to bring ever lasting impression.

To produce a unique weaving style based on African design, first individual fabrics of similar design and colors are woven in strips. Then the individual strips bound together to create the required continues woven fabrics. These methods of weaving deliberately done to produce uneven kind of individual weave; very flexible to create required color mix and intricate design. Lastly, sewers bring multitude of weave strips to a single unified design by sewing together.

\section{Designing principles}

Design is an art of putting thing around us artistically on paper and it's then transformed to fabric. This art sometimes conveys individual creativity on different circumstances. It requires the ability to arrange relate object such as lines, angles, spaces, shapes, colors, texture and other hidden values that come together to exemplify the designers abstract. The balancing principles, the movement of lines, style of determining repeating pattern, emphases and contrast hues together with unity are what make designers who they are. It is from this point Wilson said; having the idea how to design and use properly design tools for representing our own idea on paper is not enough in the design work [7, 8] but also requires understanding of African culture.

Designers understand that every elements of a single pattern has special meanings for its unity. Thus arranging and organizing these elements of pattern makes more meaningful for their work. This works can be categorized as balance, rhythm, proportion, emphases and unity. Balance is the act of putting two segments right and left to each other and maintains their equilibrium position from each other. Keeping the relative size of designed object as compared with the whole system in a given standard system goes to proportion. Rhythm is a regular arrangement of motives in a given pattern. This starts from the correct putting of lines symmetrically to each other on the other side of the design. Similarly, an emphasis is the point of focus in the drawn paper. This is sometimes called point of attention because this is the point that attracts the viewer. If the object arranged sequentially and orderly manner it will control the views attention and used as feedback for designer. Otherwise it tells us something wrong in the system. Similarly, unity is used as a check point for designers. It tells designer's whole composition, summery of all combining points as a whole and harmony of works in a design. [3, 4] 


\section{Motifs and its representation on fabric}

The concept of creating motifs and its account on African textile cloth is not only to please eyes and the viewers. But it is also used as medium of communication in a society that conveys spiritual values and beliefs from their ancestors. Bridget on his African textile study show that Africa clothes and their imported motifs define charity, culture, appropriate social relationship and their behaviors in a society $[9,10]$. Due to this deep rooted perception of cloth values in African society, design denotation has involved a lot of actors in the field and has handed over through descendants.

\section{Valuing colors and its combination for aesthetics}

Color and aesthetics are the primary task of designer to understand and justify its sensitivity in African society. Most patterns on use generally characterized by high resonance use of colors. This can be achieved through use of bold colors. There are two ways to preserve these loud colors (full sonority of colors) in African textile pattern. Firstly through using contrastive colors, hot and cool, of equal strength. Secondly by maintaining the proportionality values between lighting and dark in a given dynamics; that is rephrasing of light and dark colors until we achieve the desired mix.

Colors on fabric and cloth in the African context possess important meaning. The meaning varies from one other as we go along different ethnic clothes. Yellow represent fertility (exemplified by eggs or yoke), green stands for renewal and growth that has seen in plants by representing birth and death. The sky is blue, hence blue indicate presence of God. It refers the spirit that one keeps them in harmony. Red denotes political, power and strength while black stands for seriousness and unity. It is from this point that Bright illustrated colors on his study of "An appraisal of the aesthetics dimensions to the African philosophy of cloth” across some ethnic groups.

Generally, colors have important meanings in societies, because they might associate with history or has their own short coming. For instance, sometimes blue and grey colors are associated with cold while red and orange are linked with warm weather. Hence, colors communicate with people about the time of the day, temperature and weather of the year. Thus, estimating color and its trends are very important in the fashion industry because color play a great role with changing seasons.

\section{Materials}

Natural fibers such as cotton, silk, wool, raffia, rayon and bark of certain tries are mainly used for African fabrics. Weaving bast fibers like as jute or flax are also used for traditional fabric production. Traditionally, these fibers are prepared by hand spun method. Synthetic fibers are also introduced to increase the volume of current production.

\section{Methods to produce on Jacquard machine}

Producing the African kaleidoscope of dazzling patterns on jacquard machine gives an elegant look. These fabrics produced on contemporary weaving machines are very inspiring to watch, coupled with accuracy graced from jacquard machine. Manufacturing African fabrics such as festive headgear, bright scarves, colorful wraps, wearable art and elegant gowns of any type can be produce in more accurate ways. Manufacturing these extra-ordinary African designs to contemporary multilayered fabric would enhance its aesthetics and provides with flexible end uses.

The following cases of selected African traditional patterns have planned to exemplify the above discussion. These selected patterns are more or less elaborate kind of African design element selection, coloring technique, aesthetics and other designing principles from the following figures. 


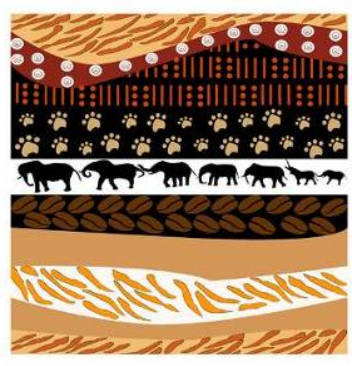

A
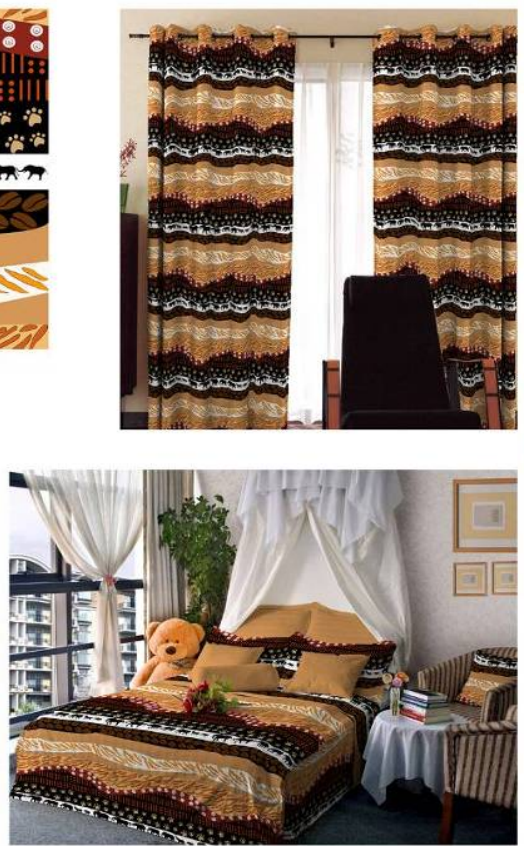
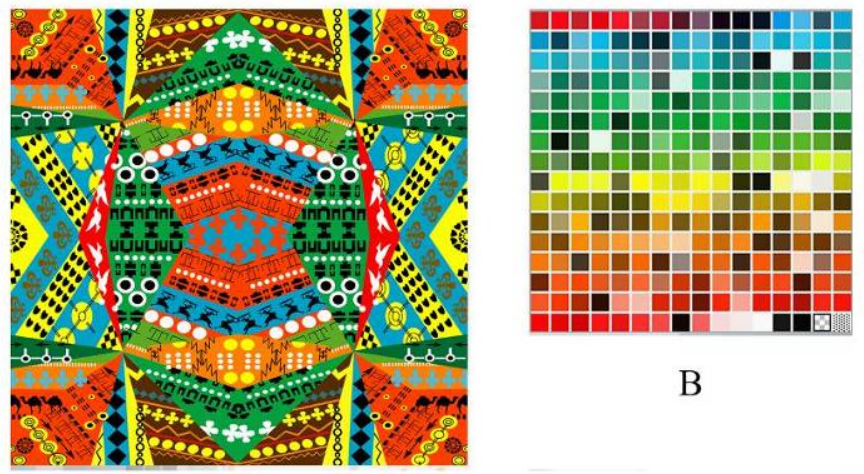

B

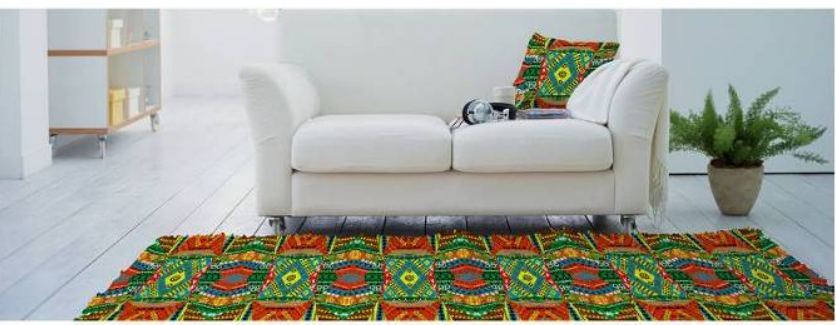

Fig. 1 African traditional patterns A) fabric designing styles and its final uses B) kaleidoscope of African style through bright colors

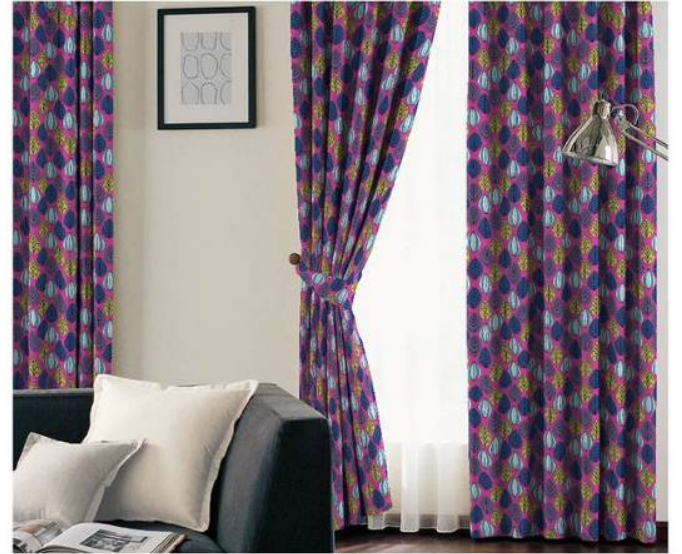

$\mathrm{C}$
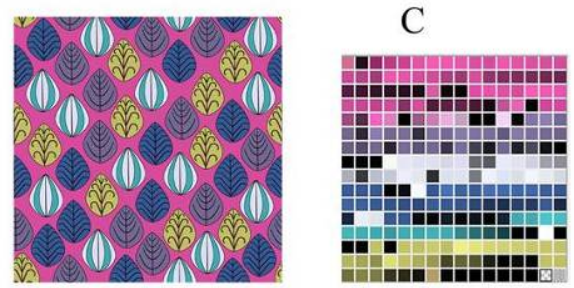
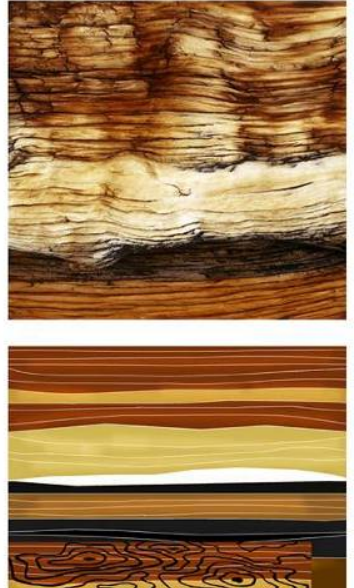

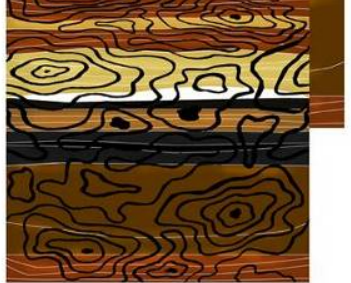

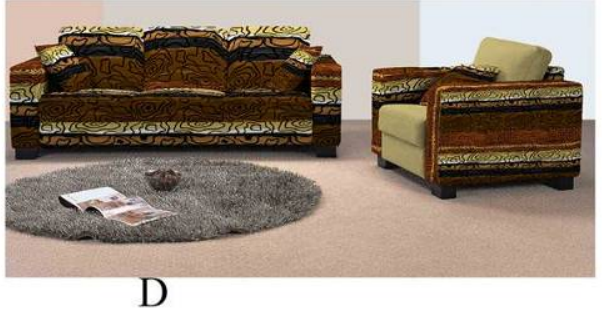

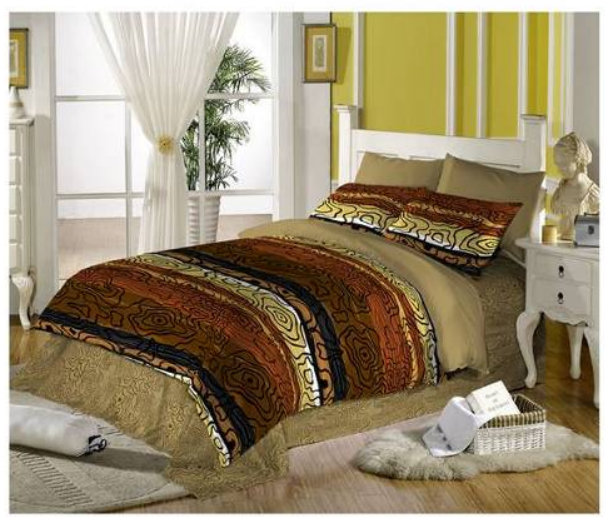

Fig. 2 C) leaf based motifs for contemporary fashion design uses D) wood patterns as source for textile design and color combinations

\section{Uses of African pattern through fabric}

Textiles in Africa have been used as a means of communication beside its formal uses. It conveys information within a particular community by referring mutual association of spirituality, historical and cultural integrity. These have been experienced for thousands of years since they were using as communication tools. The symbols and figurative compositions represent historical proverbs or events in the past. A form of storytelling, through fabric artistically preaches importance of an individual, family or in 
large social unit [5]. Commonly, due to the above secret embedded in African textiles, societies have been using for political, social and special occasions.

\section{Conclusion}

African textile and arts are means of expression that Africans use to define themselves through wearable design crafts. These textiles and arts are known throughout the world with its structure composition, color collections and with their bold size of elements across a given design are main characteristics. Every single elements of a given African pattern has special meaning and deliberately given to represent some values in a society. Those values will be embedded in a form of curves, lines or complete symbols to convey spiritual messages, hope, or other hidden values.

It is a challenge for designers to design collections embracing cultural values with limited cultural background knowledge and experiences. To avoid this problem and also to incorporate and produce outstanding patterns containing those values, designers advance their knowledge by travel to various areas of the continent to learn about different society's designs and dressing culture that has to be built in fabrics. So that many people can recognize the product as it's originated from their areas. Colors and aesthetics are one of the favorite African textiles. Colors are highly pronounced to bring loud tone by valuing two or more than two equally considered bold hues. Taking two extreme (opposite) colors like as dark and light or hot and calm of equal strength, by keeping quality of hue across a pattern are always a plan that brings viewers to focus. Generally, it is much important to consider the significance of African cultural expression on pattern besides localizing patterns on fabrics and garments as sign of originality to enhance cultural meaning.

\section{Acknowledgments}

This research was funded in part through a grant of the National Social Science Fund Art Project (12BG064) and Zhejiang key innovation team on science and technology project（2011R50004）.

\section{References}

[1] Leigh, F.P and John, 1996, the Art of African Textiles Tradition

[2] Victoria, S. 2009, Textiles and Fabric of Africa

[3] José, B. and Jennifer, R. 2007, West African Textiles Exhibit curated by assistance of Dr. Patricia Hunt-Hurst, Raúl ázquez López, and his students

[4] Tunde, M.A. 2008, the African Print Hoax, Machine Produced Textiles Jeopardize African Print Authenticity, Department of Home Science University of Agriculture, and Abeokuta, Nigeria, The Journal of Pan African Studies, vol.2, no.5

[5] Muthoni, K.2004, Fashioning Africa: Power and The politics of dress, Jean Allman, Ed. Indiana University Press, Bloomington and Indianapolis,

[6] Okougha, O.H 2010, Assessment of the acceptability of African Fabrics among Undergraduate, Department of Home Science and Management College of Food Science and human ecology, Nigeria

[8] Jacquie, W., Handbook of Textile Design Principles, Processes

[9] Bridget, O.J, Omatseye \& Kingsley, O.E 2012, an Appraisal of the Aesthetic Dimension to the African Philosophy of Cloth

[10] Emily, 2008, African textiles, Institute of Museum and Library service, New York

[11] Victoria, 2013, African Fashion from Dual-Directions: Representing Self and Other, University of Florida School of Art and Art History

[12] Xénia, F.R 2011, the language of Panos: dress and Fashion in Angola, National Museum of Costume, Lisbon 